\title{
Night Time Vehicle Detection and Classification Using Support Vector Machine
}

\author{
Prof. V. B. Sutar ${ }^{1}$, Dr. Mrs. L. S. Admuthe ${ }^{2}$ \\ ${ }^{I}$ Department of Electronics \& Telecommunication Engineering, TEI, RAJWADA, Ichalkaranji, India. \\ ${ }^{2}$ Department of Electronics Engineering, TEI, RAJWADA, Ichalkaranji. India.
}

\begin{abstract}
The paper presents a vehicle detection system by locating their headlights and tail lights in the nighttime road environment. The system detects the vehicles light in front of a micro CCD camera assisted vehicle i.e. oncoming \& preceding vehicles. Our system automatically controls vehicle's head lights status between low and high beams which avoids the glares for the drivers. The captured frames consist of number of bright objects over dark background. These objects are due to vehicle lamps, road reflection etc. The captured object features are used to train and classify the two classes of lights in vehicles light \& other light source. The machine learning based approach, Support Vector Machine (SVM) is used to accomplish this task. The output of the SVM is simply the signed distance of the test instance from the separating hyperplane. The result show the SVM is effective to classify number of lights and it is useful for vehicle validation.
\end{abstract}

Keywords - Computer vision, Driver Assistance, Image processing, Support Vector Machine, Vehicle detection.

\section{INTRODUCTION}

This paper is focused on the vehicle detection and classification in night time road driving environment. The proposed system is able to control the status of headlights between low beams and high beams. Low beams provide less illumination and are used at night to illuminate the forward path when oncoming vehicles are present. High beams provide significantly more light and are use to illuminate the vehicle's forward path when vehicles are not present $[5,7]$. There are various countries with regulations to control the amount of glare experienced by drivers due to preceding vehicles i.e. other vehicles travelling in the same direction and oncoming vehicles i.e. vehicles travelling in the opposite direction. To prevent drivers of oncoming vehicles from being subjected to excessive glare levels this is proposed. The typical night time environment is shown in Figure 1 which consists of oncoming vehicle's head lights and nuisance light sources like reflections of road or road reflectors, streetlights etc. The proposed automatic headlight control system is designed to detect both the headlights of the oncoming vehicles as well as the tail lights of preceding vehicles at night. It also distinguishes the lights between vehicles light and nuisance lights [5]. The proposed work is subdivided in, vehicles bright light detection and recognition, feature extraction and classification using support vector machine (SVM). Firstly the system captures the frames by using micro camera which is mounted in the windshield area [1, 2, 3, 4, 5, 6, 7 and 9]. Then the image processing techniques are applied on the frames to extract the features of bright object of vehicle's light. These features are used to classify the objects in vehicle's light and other light sources. This task is accomplished by a machine learning based technique, support vector machine (SVM). The classification results are used for further proposed work.

\section{PRESENT THEORY}

Chun-Che Wang et.al [1] proposed a vision-based driver assistance system to enhance the driver's safety in the nighttime. The proposed system performs both lane detection and vehicle recognition. The vehicle recognition is achieved by using an evident feature which is extracted through three four steps: taillight standing-out process, adaptive thresholding, centroid detection, and taillight pairing algorithm [2]. An automatic method is also provided to calculate the tilt and the pan of the camera by using the position of vanishing point which is detected in the image by applying canny edge detection, Hough transform, major straight line extraction and vanishing point estimation. Ming-Yang Chem et.al [2] proposed a scheme that detects the highway lane borders from the night scene. Through the brightness and area filtering in extracting the bright spots, the interference caused by vehicles and other lights can be avoided. In the latter processing with clustering algorithm and $2^{\text {nd }}$ order polynomial curve approximation, the reflector points are corrected aligned and lane border curves are quite smooth and consistent. In vehicle detection, they pair the extracted rear-lights to find the vehicles far or near. The rear-lights are extracted based on the red bright regions the white bright regions surrounded by red pixels, and the noise filtering. The elimination of those regions of improper size or position is partially based on the information of detected lane borders. In the process of rear-lights pairing they took the approach of lane partitioning to facilitate the process and use the scheme of compound rear-light to reduce the 
complexity. The pairing of rear-lights was successfully achieved. The detection of vehicles in this paper does not include the trucks and large buses, because some of their rear-lights are high above the observing camera.

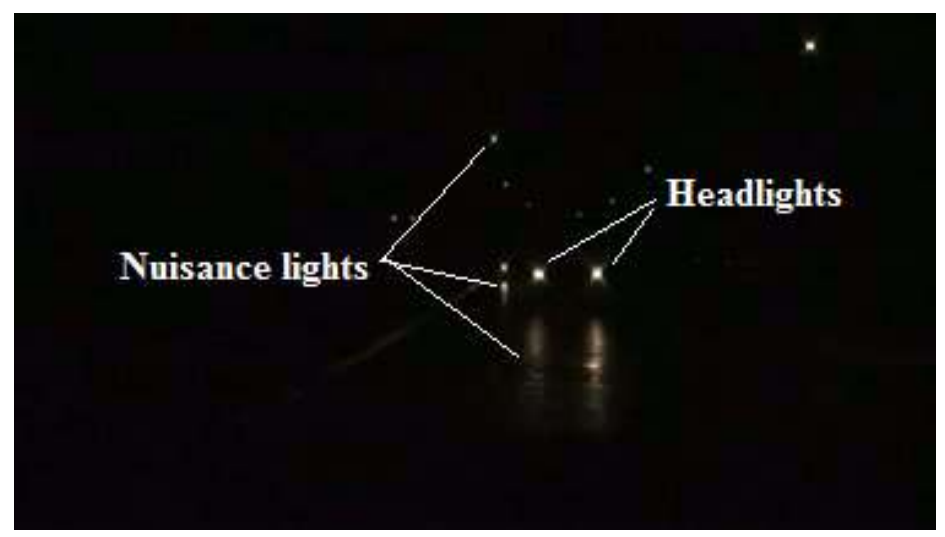

Figure 1: Typical nighttime road environment

Yi-Ming Chan et.al [3] discussed a statistical framework called particle filter is adopted to integrate the four cues including vertical edge, underneath, symmetry and the taillights. With these four steps initial sampling, propagation, observation, cure fusion and the evaluation, in the particle filtering framework, a highlevel tracking strategy with BSAS clustering technique is chosen to deal with the case that the scene is with blur image for a short period. The frame rate of proposed system was roughly $20 \mathrm{fps}$ and it was ready for real-time application. Ronan O'Malley et.al [4] have discussed the need for a system to avoid or mitigate forward collisions during night time. A background to the relevant automotive rear light legislation, showing characteristics that can be recognized by image processing, was given. They have presented an algorithm for forward collision detection at night using a visual camera [1]. The technique was able to filter red and white colours in the HSV colour space. White regions adjacent to red regions are searched for symmetrical pairs and aspect ratio constraints are applied to resulting bounding boxes. This produces detected rear target vehicle lights. P. F. Alcantarilla et.al [2, 1 and 5] presents an effective system for detecting vehicles in front of a cameraassisted vehicle i.e. preceding vehicles traveling in the same direction and oncoming vehicles traveling in the opposite direction, during night time driving conditions in order to automatically change vehicle head lights between low beams and high beams avoiding glares for the drivers. The B\&W micro-camera was mounted in the windshield area and looking at forward of the vehicle. Digital image processing techniques are applied to analyze light sources and to detect vehicles in the images. The algorithm was efficient and able to run in realtime. Andrea Fossati et.al [8] presents a system to detect and track vehicles at night, estimate their position and relative velocity and predict their trajectory. Special care has been taken to make the system robust by minimizing the false negatives and filtering out the false positives using temporal consistency. The framework was able to provide the information that can help enhancing security by warning the driver in case of potentially dangerous situations. Finally the system runs in real-time on ordinary hardware using as input a standard video camera [2, 7 and 5]. Peachanika Thammakaroon et.al [6] has proposed a predictive brake warning system in nighttime that can warn the driver for future danger by using a single camera and a speed sensor $[7,5]$. The system used an image processing algorithm that detects and measures the size of active brake lights. From the brake lights detected, the system calculated the risk of the rear-end collision from the vehicle's speed, the area of all red lights, and the largest size of the red lights. Experiment confirmed the correlation between the rear-end collision risk and the actual brakes.

Steffen Gormer et.al [7] have proposed vehicle detection system based on spot detection and pairing shows promising results and is real-time capable on a standard PC. Rough distance estimation as well as an accurate time-to-collision calculation can be done using the spots centers of gravity. A general problem for night vision applications is rain or fog on the windshield, causing light sources such as vehicle lights to blur and smear extremely [1]. Ronan O'Malley [9] have described a system that detects vehicles in front at night using a regular-color forward-facing camera also described the advantages of using standard color camera hardware as opposed to a novel or specialized platform. They have presented a camera configuration process that addresses the issues of reproducing and verifying results, portability between different camera hardware and ensuring lamp color information is not lost due to pixel saturation. Red-color thresholds have been derived from automotive regulations and adapted to real-world conditions utilizing the HSV color space, as opposed to subjective color thresholds or hardware color filters used in related research and presented a shape and sizeindependent color image cross correlation approach to pairing detected lamps. A Kalman filter [10] tracking 
algorithm has been implemented to improve robustness by ensuring continuity of operation through small detection failures and predicting future location of targets.

On-road vehicle detection, feature extraction and classification using rear-view of vehicle are presented by Zehang Sun et.al [11]. They used a Gabor filters for vehicle feature extraction and Support Vector Machines for vehicle detection. Gabor filters gives a mechanism for obtaining some degree of invariance to intensity due to global illumination, selectivity in scale, and selectivity in orientation. Vehicles do contain strong edges and lines at different orientation and scales, thus, the statistics of these features like mean, standard deviation, and skewness are assumed for vehicle detection. To provide robustness, these statistics are not extracted from the whole image but rather are collected from several sub images obtained by sub-dividing the original image into sub windows. These features are then used to train a SVM classifier. Christopher J. C. Burges et.al [12] described a linear Support Vector Machines (SVMs) for separable and non-separable data, working through a non-trivial example in detail. They described how support vector training can be practically implemented and discussed in detail the kernel mapping technique which is used to construct SVM solutions which are nonlinear in the data. They have show how Support Vector machines can have very large VC dimension by computing the VC dimension for homogeneous polynomial and Gaussian radial basis function kernels. Hyeran Byun et.al [13] presented a comprehensive survey on applications of Support Vector Machines for pattern recognition. They discussed about SVM due to good generalization performance on many real-life data and the approach is properly motivated theoretically, it has been applied to wide range of applications. They described a brief introduction of SVMs and summarize its numerous applications.

Pascal Paysan et.al [14] presented the vehicle detection using a pair of stereo cameras which are mounted in a test vehicle. The work was focused on the recognition of oncoming vehicles. As stereo vision by itself is not reliable enough to perform accurate vehicle detection it is useful to quickly generate object hypotheses which can then be verified by accurate pattern recognition techniques. They have used pattern recognition algorithm as SVM and trained on wavelet coefficients of histogram equalized frontal views of vehicles. A real-time non-intrusive fatigue monitoring system is presented by Matthew Sacco et.al [15] which exploits the driver's facial expression to detect and alert fatigued drivers. The work was consists of the ViolaJones classifier to detect the driver's facial features. The correlation coefficient template matching method was applied to derive the state of each feature on a frame by frame basis. A Support Vector Machine (SVM) was finally integrated within the system to classify the facial appearance as either fatigued or otherwise. Feature extraction and classification for rear-view vehicle detection is discussed by Zehang Sun et.al $[11,16]$. They have investigated feature extraction methods as principal component analysis, wavelets, and Gabor filters. The features are evaluated using two popular classifiers, neural networks and support vector machines (SVMs). Yulan Liang et.al [17] discussed on this strategy and applied support vector machines, which is a data mining method to develop a real time approach for detecting cognitive distraction using driver's eye movements and driving performance data. Data were collected in a simulator experiment in which ten participants interacted with an IVIS while driving. The data were used to train and test both SVM and logistic regression models.

\section{ALgORITHM}

The proposed system consist of five steps as capturing of videos, input frames form video data, thresholding and segmentation, clustering and labeling, and classification. The detailed work flow is shown in figure 3 .

1. The system captures the frames with the rate of $15 \mathrm{fps}, 20 \mathrm{fps}$ or $30 \mathrm{fps}$ and it is obtained by using micro camera in real time environment as shown in Figure 2. The pre-captured video format from camera is *avi. The input frame from pre-captured videos shows the typical nighttime road environment in front of the car. A typical nighttime road conditions are characterized by a dark background and bright objects corresponding to head lights, tail lights and nuisance light sources as shown in figure 1 . The behavior of system analyzed by considering the different aspect ratios of input frames like 376x212, 640x360, 854x480 etc. because the processing time required for small size frames is minimum than bigger size frame. The input frame format is kept *.jpeg.



Figure 2: Forward camera mounting location

2. The input frame thresholding is performed by using global single level thresholding and segmentation using canny method to segment out bright objects in the image, which are corresponded with vehicle's lights. 
3. To eliminate the unnecessary objects detected in the frame are eliminated by using morphological filtering operation.

4. The segmented objects are clustered \& labeled by using clustering algorithms, based on geometric characteristics of the objects, in order to distinguish vehicle's light or other light source.

5. In classification section the detected objects are classified in vehicle's lights and nuisance lights using support vector machine learning approach.

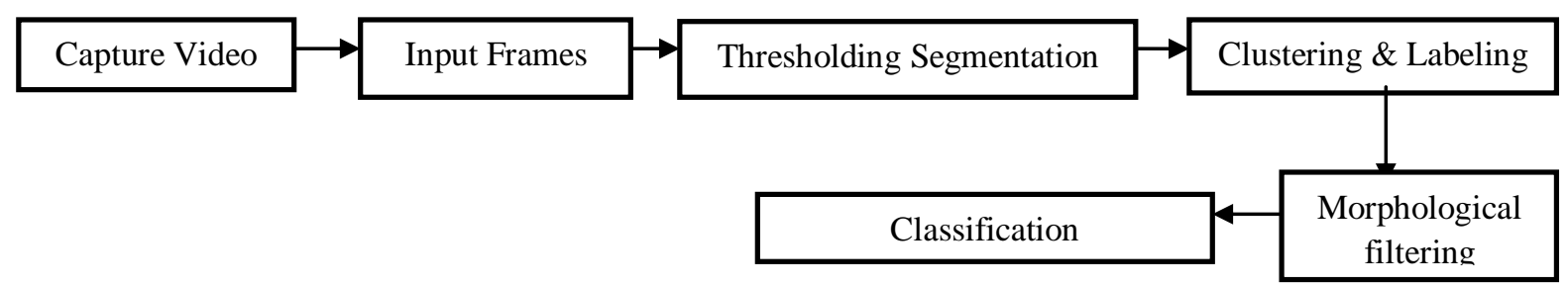

Figure 3: Functional block diagram of system

The detailed description of the proposed system is given below,

\subsection{Region of Interest (ROI)}

The captured frames are masked to create the region of interest. A region of interest (ROI) within an image is an image sub-region over which localized image processing operations can be performed. The ROI not only can reduce complexity in searching for vehicle candidates but also can decrease the false positive detection rate. We will only evaluate vehicle candidates in the region of interest. Region of Interest is created by using special algorithm with the help of masking functions to mask the unnecessary part of the frame. The algorithm returns an image sub-region defined as a binary image the same size as the original. The location of ROI is depends upon the head \& tail lamp appearance in the image as shown in figure 4.

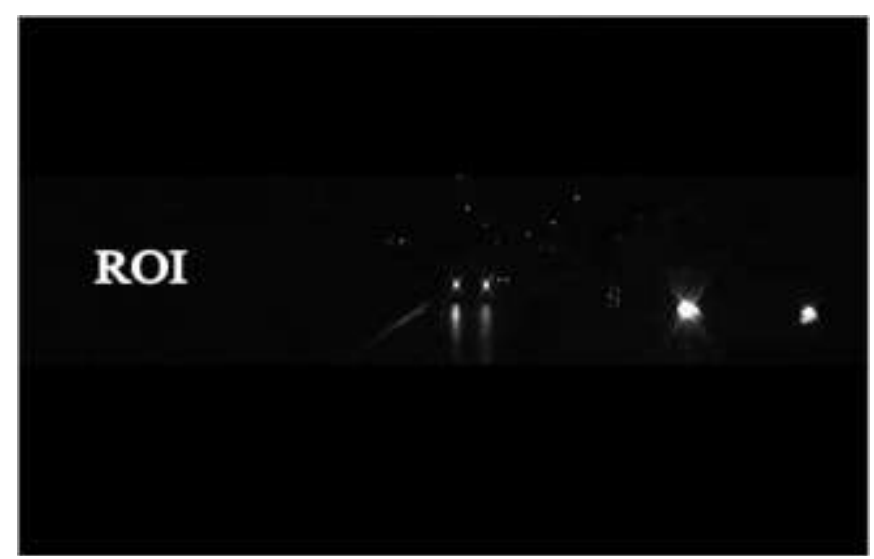

Figure 4: Intensity image with region of interest

\subsection{Bright object segmentation}

The images are thresholded using a threshold in order to detect bright objects and to measure some geometric parameters over them. Some important aspects are considered for choosing a correct threshold value such as: road illumination conditions, vehicle's lights appearance, nuisance light sources and camera parameters. To segment out the edges of bright objects canny method is used. The main reason of using this method is the edges occurring in images are not missed and that there is no response where edges do not exist. Also dual threshold is an important feature of canny. The segmented bright objects are filled by white pixels that are holes. The above thresholding creates the binary image $b(x, y)$ from an intensity image $I(x, y)$ according to following,

$$
b(x, y)=\left\{\begin{array}{l}
1 \text { if } I(x, y)>T \\
0 \text { otherwise }
\end{array}\right.
$$

Where $T$ is the thresholding level. Thresholding is obtained on region of interest i. e. masked intensity image which is done by special masking algorithm as shown in Figure 4. The image histogram of masked image is shown in Figure 5. The x-axis represents the range of values within 0-255 and y-axis shows the number of times each value actually occurs within the image. The threshold value is selected to separate the foreground of the image and vehicle's light using the thresholding. 


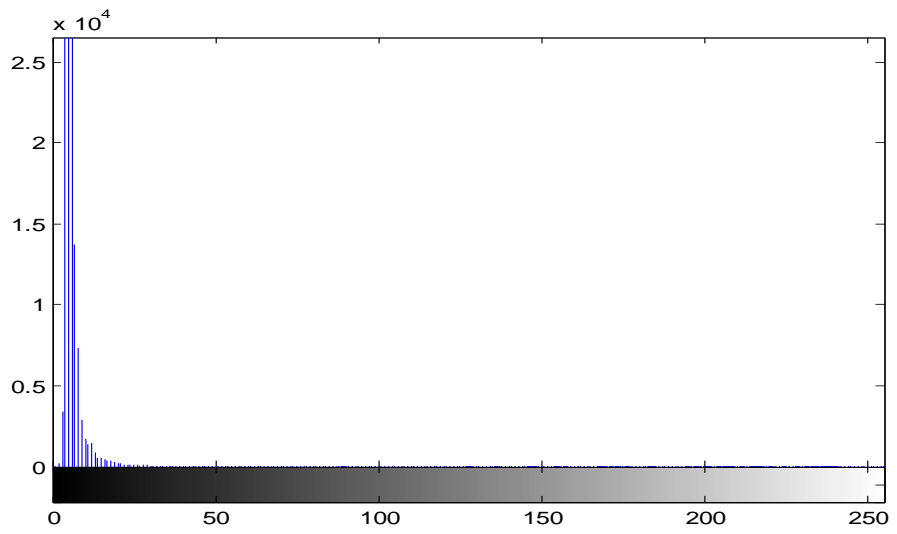

Figure 5: Histogram of masked image

Figure 6 shows the segmented image after canny method and bright object after filling of holes in segmented object is shown in Figure 7.

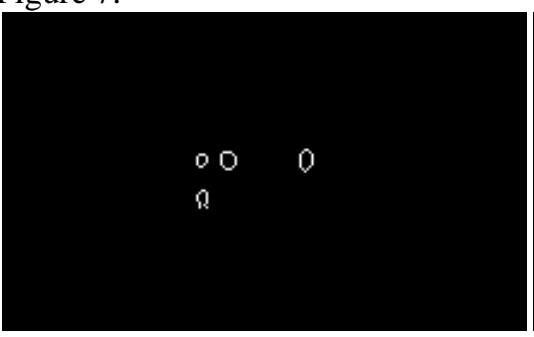

Figure 6: Edge detected image

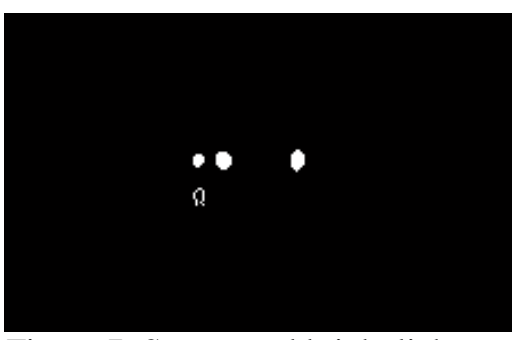

Figure 7: Segmented bright lights

\subsection{Morphological Filtering}

The non objects that are detected in frames are eliminated by using morphological functions. These functions are useful to removes objects from binary images that have fewer than ' $\mathrm{P}$ ' pixels, producing another binary image. This operation is known as an area opening. The default connectivity is 8 for two dimensional image and opening is the name given to the morphological operation of erosion followed by dilation with the same structuring element. We denote the opening of A by structuring element $B$ as,

$$
A \circ B=(A \ominus B) \oplus B \text {, }
$$

Where, $\ominus$ and $\oplus$ denote erosion and dilation, respectively. Opening removes small objects from the foreground (usually taken as the dark pixels) of an image, placing them in the background. Figure 8 shows the image after thresholding and segmentation, which includes two head lights and some fake lights. After elimination of fake lights figure 9 shows totally clear image which consists only two blobs of head light.
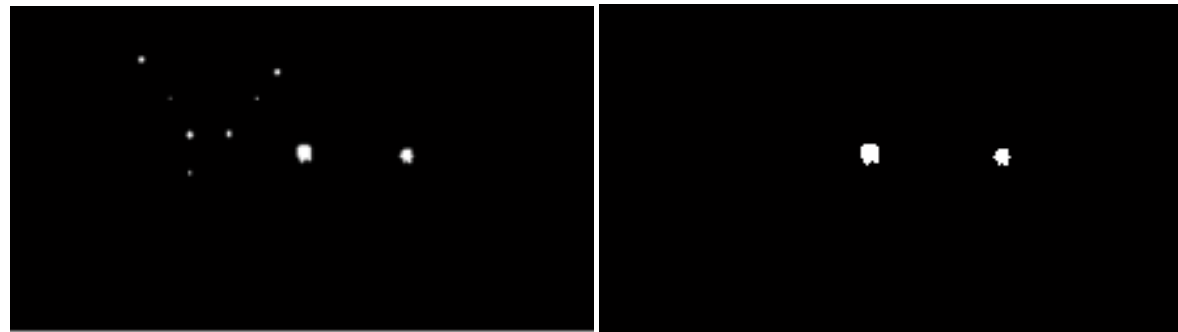

Fig. (8): Segmented image

Fig. (9): Morphologically filtered image

\subsection{Clustering and labeling}

The goal of this process is to cluster the detected blobs in the previous step and attach a label to each observation or data point in a set which are close to each other. It extracts the connected components and leads to new image. New image with connected group of pixels are sequential assigned with integer values like 0 for dark background, 1 for first detected object, 2 for second detected object and so on. Once the objects that are detected in frame \& get labelled as in figure 10 then we can estimate the feature of object according to shape of head light, tail light, road reflection, vehicle bumper reflections etc. The features like area, bounding box are shown in table 1 as per clustered object. For example the area of object number 2 and 3 is approximately equal i. e. 12 and 11 respectively as compared to object number 1 . These features are important for classification of vehicle's light and nuisance light because there is a large variation between them. 


\begin{tabular}{|c|c|c|}
\hline $\begin{array}{c}\text { Clustered } \\
\text { object }\end{array}$ & Area & Bounding Box Parameters \\
\hline 1 & 02 & $\mathrm{X}=300, \mathrm{Y}=230, \mathrm{~W}=02, \mathrm{H}=01$ \\
\hline 2 & 12 & $\mathrm{X}=392, \mathrm{Y}=240, \mathrm{~W}=06, \mathrm{H}=06$ \\
\hline 3 & 11 & $\mathrm{X}=442, \mathrm{Y}=241, \mathrm{~W}=06, \mathrm{H}=06$ \\
\hline
\end{tabular}

Table 1: Area and Bounding box

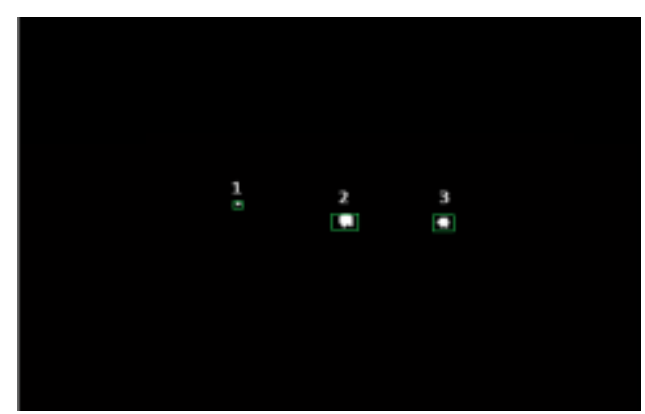

Fig. (10): Labeled image

\subsection{Classification}

The critical problem of the system is to distinguish between vehicle's lights and reflections of traffic signs (nuisance light source). The detected bright objects are classified as nuisance or vehicle's light depending on the area and aspect ratio because it is always equal for vehicle's light as compared to nuisance light sources. There are two essential aspects of SVMs classifiers as the training strategy and the classifier structure. As SVMs are supervised learning methods used for classification, it is necessary to obtain a model under supervised training and once the model is trained, it can be used in real applications.

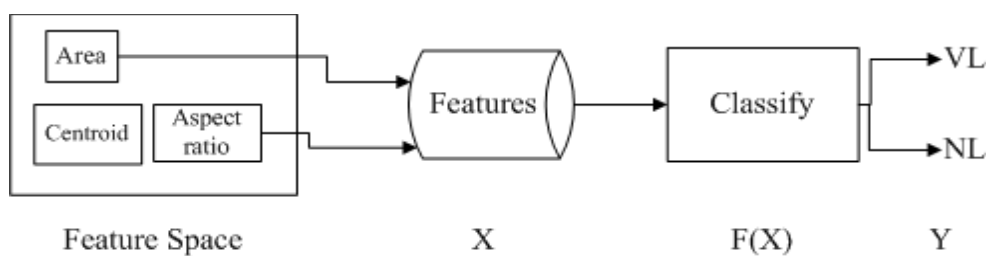

Fig. (11): Classification process

Figure 11 shows the classification process where an input vector is defined for the classifier which is composed of different parameters. These parameters are computed per object and define the state vector for the SVM. The parameters of the vector like Area in pixels, Coordinates of the object's centroid, Aspect Ratio. The output of SVM is simply the signed distance of the test instance from the separating hyperplane. This output indicates whether the analyzed object corresponds to a vehicle's light or nuisance light.

The classification algorithm uses this result and classifies the objects as nuisance or vehicles depending on its output distance from the separating hyperplane. If $k$ is number of training patterns, $\left(X_{1}, X_{2}, \ldots ., X k\right) \&$ their labels $\left(Y_{1}, Y_{2}, \ldots, Y_{k}\right)$.

$$
X \in R^{n}, Y \in\{+1,-1\}
$$

Where, $R^{n}$ is real n-dimensional vector space. In our analysis the vector space consists of two classes of feature area and aspect ratio. Support vector machine constructs a separating hyperplane $\mathrm{g}(\mathrm{x})=0$ that will acts as decision surface as shown in figure 12 .

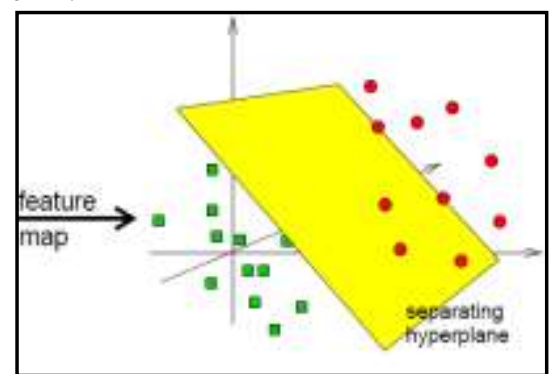

Fig. (12): Construction of separating hyperplane 
The decision function $d(\mathrm{x}, \mathrm{w}, \mathrm{b})$ consists the parameter weight vector $\mathrm{w}=\left[w_{l}, w_{2}, \ldots, w_{n}\right]^{\mathrm{T}}$ and bias $b$ which is given by,

$$
d(x, w, b)=w^{T} x+b=\sum_{i=1}^{n} w_{i} x_{i}+b
$$

After training is completed, using the weight vector $w$ and the bias $b$, a pattern $x$ is classified as by examining the sign of $d(x, w, b)$.

If $d(x p, w, b)>=0$, pattern xp belongs to class $1,(\mathrm{yp}=1)$

If $d(x p, w, b)<0$, pattern xp belongs to class $2,(\mathrm{yp}=-1)$

The equation $w x^{T}+b=0$ divides the input space into two half spaces $\left(R^{2}\right)$.

Thus the output of support vector machine is simply the signed distance of the test instance from the separating hyperplane. The output shows whether the analyzed object corresponds to a vehicle or not. The classification algorithm uses this result and classifies the objects as vehicles and nuisance light depending on its output distance from the separating hyperplane.

\section{EXPERIMENTAL RESULTS}

The proposed system is analyzed by using different sizes of the recorded image sequences like $376 \times 212,640 \times 360,854 \times 480$ and $640 \times 480$ pixels per frame so as to detect vehicles at far distances. The computation time spent on processing one input frame depends on the complexity of the road scene. The experiment on algorithm is carried out by considering different frame rates like $15 \mathrm{fps}, 25 \mathrm{fps}$, and $30 \mathrm{fps}$. The frame rate of the system is in average close to $20 \mathrm{fps}$ which is enough for real-time demands. The summary of detected number of lights is given in following tables.

Table 2: Tail light detection results

\begin{tabular}{|c|c|c|c|c|c|c|}
\hline $\begin{array}{c}\text { No. of } \\
\text { Sequence }\end{array}$ & $\begin{array}{c}\text { Number of } \\
\text { Frames }\end{array}$ & Frame Size & $\begin{array}{c}\text { Frame Rate } \\
\text { (fps) }\end{array}$ & $\begin{array}{c}\text { Classified } \\
\text { Tail Lights }\end{array}$ & $\begin{array}{c}\text { Classified Nuisance } \\
\text { Lights }\end{array}$ & $\begin{array}{c}\text { Total No. } \\
\text { of Lights }\end{array}$ \\
\hline 1 & 102 & $480 \times 360$ & 30 & 05 & 12 & 17 \\
\hline 2 & 093 & $480 \times 360$ & 30 & 02 & 02 & 04 \\
\hline 3 & 340 & $640 \times 360$ & 30 & 02 & 04 & 06 \\
\hline 4 & 367 & $640 \times 360$ & 30 & 02 & 00 & 02 \\
\hline 5 & 267 & $376 \times 212$ & 15 & 02 & 01 & 03 \\
\hline
\end{tabular}

Table 2 shows the tail light detection results of algorithm which includes the classified vehicle's tail light count and nuisance light count. In the same way table 3 shows the detailed results of head light detection. Figure 14 shows trained \& classified patterns for sequence 5 in table 2 with maximal margin of separation. The performance of classifier for this training \& classification is 1 . The generalization performance of classifier is depends on the maximal margin of separation between the two classes. Figure 14 shows the training \& classification for tail light with maximal margin with two classes and two features. The red plus marks are training patterns and pink plus marks are classified patterns in tail light class. The nuisance light class consists of green plus marks of training patterns and cyan plus marks of classified patterns. Figure 13 shows the result of SVM for head light classification for first sequence in table 3 which is based on feature area and aspect ratio.

Table 2: Head light detection results

\begin{tabular}{|c|c|c|c|c|c|c|}
\hline $\begin{array}{c}\text { No. of } \\
\text { Sequence }\end{array}$ & $\begin{array}{c}\text { Number of } \\
\text { Frames }\end{array}$ & Frame Size & $\begin{array}{c}\text { Frame Rate } \\
\text { (fps) }\end{array}$ & $\begin{array}{c}\text { Classified } \\
\text { Tail Lights }\end{array}$ & $\begin{array}{c}\text { Classified Nuisance } \\
\text { Lights }\end{array}$ & $\begin{array}{c}\text { Total No. } \\
\text { of Lights }\end{array}$ \\
\hline 1 & 191 & $640 \times 480$ & 25 & 2 & 3 & 5 \\
\hline 2 & 191 & $376 \times 212$ & 15 & 2 & 2 & 5 \\
\hline 3 & 638 & $376 \times 212$ & 15 & 2 & 0 & 2 \\
\hline 4 & 320 & $376 \times 212$ & 15 & 4 & 0 & 4 \\
\hline 5 & 890 & $376 \times 212$ & 15 & 3 & 5 & 8 \\
\hline 6 & 230 & $640 \times 360$ & 30 & 3 & 2 & 5 \\
\hline 7 & 100 & $640 \times 360$ & 30 & 3 & 5 & 8 \\
\hline 8 & 70 & $640 \times 360$ & 30 & 2 & 5 & 7 \\
\hline 9 & 147 & $640 \times 360$ & 30 & 2 & 9 & 11 \\
\hline 10 & 380 & $854 \times 480$ & 30 & 2 & 0 & 2 \\
\hline 11 & 160 & $854 \times 480$ & 30 & 2 & 1 & 3 \\
\hline
\end{tabular}






Fig. (13): Classification of head light \& nuisance light

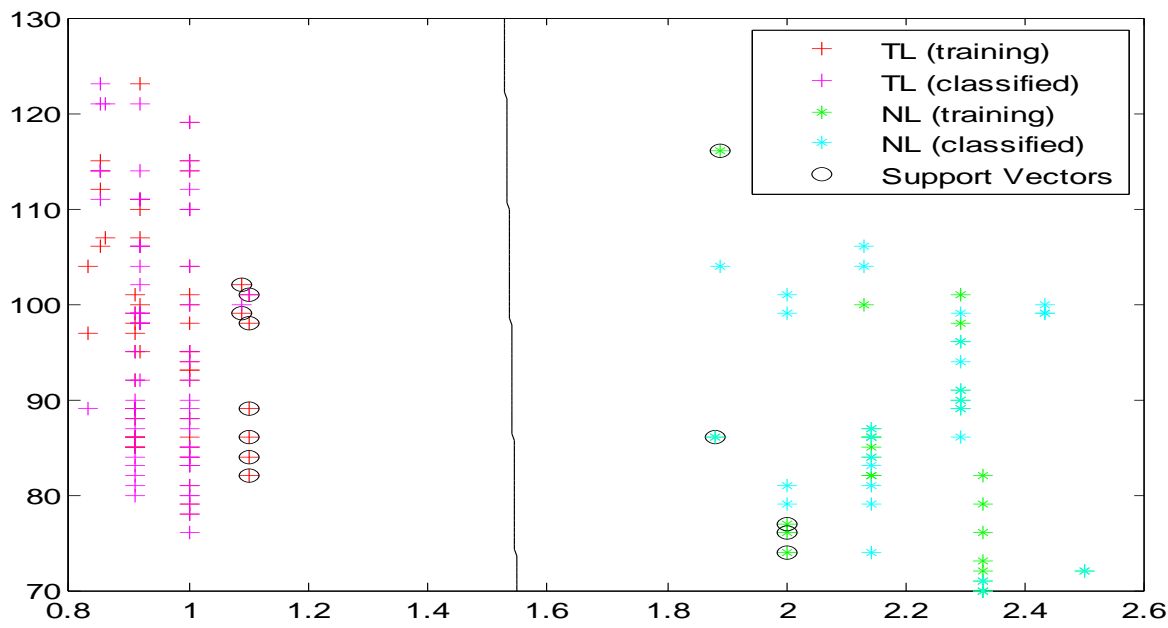

Fig. (14): Classification of tail light \& nuisance light

\section{CONCLUSION AND FUTURE SCOPE}

We have presented a night time vehicle rendition and classification system for automatic beam control. The detection algorithm extracts the bright objects at night using thresholding and segmentation approach and estimated different features. The features of detected bright object like area, aspect ratio, symmetry, distance are used to train the classifier in order to classify the objects between head light, tail light and nuisance lights. The $100 \%$ detection results with negligible false positives are obtained from detection algorithm. Machine learning based approach, SVM is proposed for classification and it is observed that the acceptable classification results. The obtained results demonstrate that the proposed system is effective and offers advantages for vehicle detection, classification, beam status control and collision warning.

Thus we are planning to include several improvements in detection and classification section of current proposed work. To increase the accuracy of the classifier more work must be done in algorithm. To increase the tail light detection rang more than $200 \mathrm{~m}$ to $300 \mathrm{~m}$ we are going to introduce a red filter into the system.

\section{REFERENCES}

[1] Chun-Che Wang, Shih-Shinh Huang , Li-Chen Fu, Pei-Yun Hsiao, National Taiwan University, Taipei, Taiwan, R.O.C., Driver Assistance System for Lane Detection and Vehicle Recognition with Night Vision, IEEE Transactions on Intelligent Transportation Systems, Vol. 3, No. 3, pp 203-209, Sept. 2002.

[2] Ming-Yang Chem, Ping-Cheng Hou, National Chung Cheng University, Min-Hsiung, Chia-Yi, Taiwan, The Lane Recognition and Vehicle Detection at Night for A Camera-Assisted Car on Highway, Proceedings of the 1003 IEEE, International Conference on Robotics \& Automation, Taipei. Taiwan, September 14-19, 2003.

[3] Yi-Ming Chan, Shih-Shinh Huang, Member, IEEE, Li-Chen Fu, Fellow, IEEE, and Pei Yung Hsiao, Member, IEEE, Vehicle Detection Under Various Lighting Conditions by Incorporating Particle Filter, Proceedings of the 2007 IEEE Intelligent Transportation Systems Conference Seattle, WA, USA, Sept. 30 - Oct. 3, 2007. 
[4] O'malley R. Glavin, M. and Jones. Connaught Automotive Research Group Department of Electronic Engineering, National University of Ireland, Galway, Vehicle Detection at Night Based on Tail-Light Detection, ISVCS. ${ }^{\text {st }}$ International ICST Symposium on Vehicular Computing Systems. ISVCS2008.3546, E. 2008.

[5] P. F. Alcantarilla, L.M. Bergasa, P. Jim'enez, M. A. Sotelo, I. Parra, D. Fernandez, Department of Electronics. University of Alcal'a, Alcal'a de Henares (Madrid), Spain, Night Time Vehicle Detection for Driving Assistance LightBeam Controller, 2008 IEEE Transaction, Eindhoven University of Technology Eindhoven, The Netherlands, June 4 -6, 2008.

[6] Peachanika Thammakaroon, Poj Tangamchit Department of Control System and Instrumentation Engineering, Kmg Mongkut's University of Technology Thonburi, Thailand, Predictive Brake Warning at Night using Taillight Characteristic, IEEE International Symposium on Industrial Electronics (IS1E 2009).

[7] Steffen Gormer, Dennis Muller, Stephanie Hold, Faculty of Electrical Engineering and Media Technologies, University of Wuppertal, D-42119 Wuppertal, Germany. Mirko Meuter, and Anton Kummert, Delphi Electronics \& Safety Advanced Engineering D-42119 Wuppertal, Germany. Vehicle Recognition and TTC Estimation at Night based on Spotlight Pairing, Proceedings of the 12th International IEEE Conference on Intelligent Transportation Systems, St. Louis, MO, USA, October 3-7, 2009.

[8] Andrea Fossati, CV Lab - EPFL 1015 Lausanne-Switzerland, Patrick Schonmann Cinetis SA 1920 Martigny - Switzerland, Pascal Fua CV Lab - EPFL 1015 Lausanne- Switzerland, Real-Time Vehicle Tracking for Driving Assistance, Machine Vision and Applications (ISSN: 1432-1769), 24 Jun 2010.

[9] Ronan O'Malley, Edward Jones, Member, IEEE, and Martin Glavin, Member, IEEE, Rear-Lamp Vehicle Detection and Tracking in Low-Exposure Color Video for Night Conditions, IEEE Transactions on intelligent transportation systems, vol. 11, no. 2, June 2010 .

[10] M. Betke, E. Haritaoglu, and L. S. Davis, Real-time multiple vehicle detection and tracking from a moving vehicle, Mach. Vis. Appl., Vol. 12, 2000, pp. 69-83.

[11] Zehang Sun, George Bebis and Ronald Miller, Computer Vision Laboratory, Department of Computer Science, University of Nevada, Reno e-Technology Department, Ford Motor Company, Dearborn, MI, On-road vehicle detection using gabor filters and Support vector machines.

[12] Christopher j.c. burges, Bell Laboratories, Lucent Technologies, A Tutorial on Support Vector Machines for Pattern Recognition, Data Mining and Knowledge Discovery, 2, 121-167 (1998), Kluwer Academic Publishers, Boston. Manufactured in The Netherlands.

[13] Hyeran Byun and Seong-Whan Lee, Department of Computer Science, Yonsei University Shinchon-dong, Seodaemun-gu, Seoul 120-749, Korea, Applications of Support Vector Machines for Pattern Recognition: A Survey, S.-W. Lee and A. Verri (Eds.): SVM 2002, LNCS 2388, pp. 213-236, 2002. Springer-Verlag Berlin Heidelberg 2002.

[14] Pascal Paysan, Stereovision based vehicle classification using support vector Machines, University of Applied Sciences Fachhochschule Esslingen, February 28, 2004.

[15] Matthew Sacco, Reuben A. Farrugia, Department of Communications and Computer Engineering University of Malta, Driver Fatigue Monitoring System Using Support Vector Machines, Msida, MSD 2080, Malta.

[16] Zehang Sun, George Bebis, and Ronald Miller, Monocular Precrash Vehicle Detection: Features and Classifiers, IEEE Transactions On Image Processing, Vol. 15, No. 7, July 2006.

[17] Yulan Liang, Michelle L. Reyes, and John D. Lee, Real-Time Detection of Driver Cognitive Distraction Using Support Vector Machines, University of Iowa, Iowa City, IA 52242 USA, IEEE Transactions On Intelligent Transportation Systems, Vol. 8, No. 2, June 2007 